\title{
The Wightman Axioms for the Weakly Coupled Yukawa Model in Two Dimensions
}

\author{
J. Magnen` and R. Seneor*
}

ZiF, University of Bielefeld, D-4800 Bielefeld 1, Federal Republic of Germany

\begin{abstract}
We prove the convergence of a cluster expansion for the weakly coupled Yukawa model in two dimensions.
\end{abstract}

\section{Introduction and Results}

The purpose of this paper is to prove the convergence of a cluster expansion $[8,3]$ for the Yukawa model in two dimensions ${ }^{1}$. We use here the model as defined by Seiler [13] and McBryan [10], and we shall use the presentation of Seiler and Simon [14].

The Yukawa model has been also studied by Glimm [4], Glimm and Jaffe [5] and [6], Schrader [12], Brydges and Federbush [2] and Brydges [1].

In this introduction we define the problem and state the main results, in the second chapter we define and give the properties of our main tool: a set of decoupling functions allowing to do the cluster expansion-see also [9]-, in the last chapter we prove the convergence of the cluster expansion.

Let us give some definitions, see [14].

The partition function in a volume $\Lambda$ is:

$$
Z_{A}=\int d \mu \operatorname{det}_{\text {ren }}\left(1+K_{\Lambda}\right) \text {. }
$$

The unnormalized Schwinger functions in a volume $\Lambda$ are:

$$
\begin{aligned}
& S_{\Lambda}\left(f_{1}, \ldots, f_{n} ; g_{1}, \ldots, g_{N} ; h_{1}, \ldots, h_{N}\right) \\
& =\int d \mu\left\{\operatorname{det}_{i k} S_{F}\left(\left(P^{2}+m^{2}\right)^{-1 / 4} g_{i}, \frac{P+m}{\left(P^{2}+m^{2}\right)^{3 / 4}} h_{k}\right)\right\} \prod_{l=1}^{n} \varphi\left(f_{l}\right) \operatorname{det}_{\text {ren }}\left(1+K_{\Lambda}\right)
\end{aligned}
$$

where:

$$
S_{F}\left(g^{\prime}, h^{\prime}\right)=\left(g^{\prime}, \frac{1}{1+K_{\Lambda}} h^{\prime}\right)_{L^{2}}
$$

* On leave of absence from Centre de Physique Théorique, Ecole Polytechnique, F-91120 Palaiseau, France

1 A. Cooper and L. Rosen have shown also the same result [17] 
and $i P$ is the gradient operator $K_{A}$ is the operator in $L^{2}$ of kernel:

$$
\begin{aligned}
& K_{\Lambda}(x, y) \\
& =\left\{\int \lambda\left[\left(P^{2}+m^{2}\right)^{-1 / 4} \frac{P+m}{\left(P^{2}+m^{2}\right)^{1 / 2}}\right](x, z) \Gamma \phi(z) \Lambda(z)\left[P^{2}+m^{2}\right]^{-1 / 4}(z, y) d z\right\} .
\end{aligned}
$$

$\lambda$ is the coupling constant and is real, $m$ is the mass of the fermion, $\Gamma=i \gamma_{5}$ in the pseudoscalar case and $\Gamma=1$ in the scalar case, $\Lambda(x)$ is the characteristic function of the volume $\Lambda$.

Also:

$$
\operatorname{det}_{\text {ren }}\left(1+K_{\Lambda}\right)=\operatorname{det}_{3}\left(1+K_{\Lambda}\right) B
$$

with

$$
\operatorname{det}_{p}(1+K):=\operatorname{det}(1+K) \exp \left(\sum_{n=1}^{p-1}(-1)^{n} \frac{1}{n} \operatorname{Tr} K^{n}\right)
$$

and

$$
B:=\exp \left\{-\frac{M^{2}}{2} \int: \phi^{2}:(x) \Lambda(x) d x-\frac{1}{2} \operatorname{Tr}_{\mathrm{reg}}: K_{\Lambda}^{2}:\right\} .
$$

$\operatorname{Tr}_{\mathrm{reg}}: K_{\Lambda}^{2}$ : is defined as in [14] and we note:

$\operatorname{Tr}_{\mathrm{reg}}: K_{\Lambda}^{2}:=\int d x d y: \phi(x) b_{\mathrm{reg}}(x, y) \phi(y):$.

Also $d \mu$ is the Gaussian measure of mean zero and covariance

$$
C_{\bar{m}}(x, y)=\frac{1}{(2 \pi)^{2}} \int \frac{e^{i p(x-y)}}{p^{2}+\bar{m}^{2}} d^{2} p .
$$

Finally $f_{l}, g_{j}, h_{k}$ are functions in suitable spaces (defined later), and for convenience we suppose that their supports are localized in unit squares of a lattice cover of $R^{2}$ :

$$
R^{2}=\bigcup_{\alpha \in z^{2}} \Delta_{\alpha}, \quad \Delta_{\alpha} \text { is the unit square centered at } \alpha .
$$

Let:

$$
\begin{aligned}
& \hat{g}_{i}^{\alpha}(x)=\left[\left(P^{2}+m^{2}\right)^{-1 / 4} g_{i}\right](x) \chi_{\Delta_{\alpha}}(x) \\
& \hat{h}_{k}^{\alpha}(x)=\left[\left(\frac{P+m}{\left.P^{2}+m^{2}\right)^{3 / 4}}\right) h_{k}\right](x) \chi_{\Delta_{\alpha}}(x)
\end{aligned}
$$

where $\chi_{\Delta}$ is the characteristic function of the unit square $\Delta$. Then:

$$
S_{\Lambda}=\sum_{\alpha_{l}, \beta_{k}} \tilde{S}_{\Lambda}\left(f_{1}, \ldots, f_{n} ; \hat{g}_{1}^{\alpha_{1}}, \ldots, \hat{g}_{N}^{\alpha_{N}} ; \hat{h}_{1}^{\beta_{1}}, \ldots, \hat{h}_{N}^{\beta_{N}}\right)
$$

with

$$
\begin{aligned}
& \tilde{S}_{\Lambda}\left(f_{1}, \ldots, f_{n} ; \hat{g}_{1}, \ldots, \hat{g}_{N} ; \hat{h}_{1}, \ldots, \hat{h}_{N}\right) \\
& :=\int d \mu \operatorname{det}_{i k} S_{F}\left(\hat{g}_{i}, \hat{h}_{k}\right) \prod_{l=1}^{n} \phi\left(f_{1}\right) \operatorname{det}_{\mathrm{ren}}\left(1+K_{\Lambda}\right) .
\end{aligned}
$$


We construct the cluster expansion for $\tilde{S}_{A}$ (all the functions, $f, \hat{g}, \hat{h}$ having their support in unit squares); $S_{A}$ is obtained by resummation.

Remark. In the sequel we shall take the boson mass $\bar{m}$ to be equal to $m$, because in the analysis of the convergence of the cluster expansion the two masses play the same role.

Let us call $Z^{2 *}$ the sides of the squares in the net defined by the lattice cover of $R^{2}$. To each $b \in Z^{2^{*}}$ is associated a variable $s_{b}, 0 \leqq s_{b} \leqq 1$. For each choice of $\left\{s_{b}\right\}_{b \in Z^{2}}$ We define $s$-dependant quantities:

$$
\begin{aligned}
C(s)(x, y) & =\sum_{\alpha, \beta, \gamma \in Z^{2}} H\left(s ; \Delta_{\alpha}, \Delta_{\beta}, \Delta_{\gamma}\right) M\left(\Delta_{\alpha}, \Delta_{\beta}, \Delta_{\gamma}\right) C_{m}(x, y) \chi_{\Delta_{\alpha}}(x) \chi_{\Delta_{\beta}}(y) \\
K(s)_{\Lambda}(x, y) & =\sum_{\alpha, \beta, \gamma \in Z^{2}} H\left(s ; \Delta_{\alpha}, \Delta_{\beta}, \Delta_{\gamma}\right) K_{\Delta_{\gamma} \cap \Lambda}(x, y) \chi_{\Delta_{\alpha}}(x) \chi_{\Delta_{\beta}}(y) .
\end{aligned}
$$

The definition of $b_{\text {reg }}(s)(x, y)$ follows in a natural way from the definition of $K(s)$.

Then if in formula (I.1) and (I.2) we replace all the quantities by the corresponding $s$-dependant quantities this defines $\tilde{S}_{(s)}$ and $Z_{(s)}$.

In Chapter II we define $H\left(s ; \Delta_{\alpha}, \Delta_{\beta}, \Delta_{\gamma}\right)$ and $E\left(\Delta_{\alpha}, \Delta_{\beta}, \Delta_{\gamma}\right)$ and prove:

Lemma I.1. $0 \leqq H\left(s ; \Delta_{\alpha}, \Delta_{\beta}, \Delta_{\gamma}\right) \leqq 1,0 \leqq M\left(\Delta_{\alpha}, \Delta_{\beta}, \Delta_{\gamma}\right) \leqq 1$,

$$
\sum_{\gamma} M\left(\Delta_{\alpha}, \Delta_{\beta}, \Delta_{\gamma}\right)=1 \text {. }
$$

If $s_{b}=1 \forall b$ then $H\left(s ; \Delta_{\alpha}, \Delta_{\beta}, \Delta_{\gamma}\right) \equiv 1$.

Let $D \subset Z^{2 *}$ defined by $D=\left\{b \in Z^{2 *} \mid s_{b}=0\right\}$ and let $R^{2} \backslash D=X_{1} U \ldots U X_{p}$ be the decomposition of $R^{2} \backslash D$ as a disjoint union of connected components then:

$$
H\left(s ; \Delta_{\alpha}, \Delta_{\beta}, \Delta_{\gamma}\right)=\left.\sum_{k} H\left(s ; \Delta_{\alpha}, \Delta_{\beta}, \Delta_{\gamma}\right)\right|_{X_{k}}
$$

where

$$
\left.H\left(s ; \Delta_{\alpha}, \Delta_{\beta}, \Delta_{\gamma}\right)\right|_{X_{k}}=\left\{\begin{array}{l}
H\left(s ; \Delta_{\alpha}, \Delta_{\beta}, \Delta_{\gamma}\right) \\
0 \text { otherwise. }
\end{array} \text { if } \Delta_{\alpha} \subset X_{k}, \Delta_{\beta} \subset X_{k}, \Delta_{\gamma} \subset X_{k}\right.
$$

It is then clear that $C(s)(x, y)=\left.\bigoplus_{k} C(s)(x, y)\right|_{X_{k}}$ and also that $K(s)_{\Lambda}=\left.\sum_{k} K(s)\right|_{X_{k}}$ [where $\left.K(s)\right|_{X_{k}}$ means the restriction of $K(s)$ to $\left.L^{2}\left(X_{k}\right)\right]$.

Then

$$
\frac{1}{1+K(s)}=\prod_{k} \frac{1}{1+\left.K(s)\right|_{X_{k}}} \text { and } \operatorname{det}(1+H(s))=\prod_{k} \operatorname{det}\left(1+\left.K(s)\right|_{X_{k}}\right)
$$

and this is obviously true also for $\operatorname{det}_{\text {ren }}(1+K(s))$ because $\operatorname{Tr} K^{q}$ decomposes itself in a sum $\sum_{k} \operatorname{Tr}\left(\left.K\right|_{X_{k}}\right)^{q}$.

Also

$$
\operatorname{det}_{i k} S_{F}\left(\hat{g}_{i}, \hat{h}_{k}\right)= \pm \prod_{k} \operatorname{det}_{j l} S_{F}\left(\hat{g}_{j}, \hat{h}_{l}\right)
$$

where for $k$ given the determinant is formed with the function:

$\operatorname{supp} \hat{g}_{j} \subset X_{k}, \quad \operatorname{supp} \hat{h}_{l} \subset X_{k}$. 
Then with obvious notation we have:

$$
Z=\prod_{k} Z_{X_{k}} \quad \tilde{S}=\left.\prod_{X_{k}} \tilde{S}\right|_{X_{k}} .
$$

As a consequence, according to the general scheme of the cluster expansion (see [8] and [3]), one has a convergent cluster expansion if one can prove that for some values of the parameters $\lambda$ and $m(m \geqq 1)$ :

$\left.A_{1}\right) \Delta$ being a unit square. $Z_{(\mathrm{s}), \Delta}>0$ for $s_{b}=0 b \in \partial \Delta$.

$\left.A_{2}\right)$ Let $\Gamma \subset Z^{2 *}$ and $\partial^{\Gamma}$ note $\prod_{b \in \Gamma} \frac{d}{d s_{b}}$. Let $X$ be one of the connected components of $R^{2} \backslash\left(Z^{2 *} \Gamma\right)$ then:

$$
\begin{aligned}
& \left|\partial^{\Gamma} \tilde{S}_{(s), \Lambda}\left(f_{1}, f_{n} ; \hat{g}_{1}, \ldots, \hat{g}_{N} ; \hat{h}_{1}, \ldots, \hat{h}_{N}\right)\right| \\
& \leqq \prod_{i=1}^{n}\left\|f_{i}\right\|_{-1} \prod_{j=1}^{N}\left\|\hat{g}_{j}\right\|_{L_{2}}\left\|\hat{h}_{j}\right\|_{L_{2}} O(1)^{N} O(1)^{n} \prod_{\Delta}(n(\Delta) !)^{1 / 2} e^{-Q_{1}|\Gamma|}
\end{aligned}
$$

where $|\Gamma|$ is the number of bonds in $\Gamma,\|f\|_{-1}^{2}=\int \frac{|\tilde{f}(p)|^{2}}{\left(p^{2}+1\right)} d p, n(\Delta)$ is the number of $f_{i}$ with support in $\Delta$ and $Q_{1}$ is some positive constant large enough. Also for a matrix $|A|=\sup _{i, j}\left|A_{i j}\right|$.

Indeed, giving us $\lambda_{0}>0$, there exists $m\left(\lambda_{0}\right)$ such that for $|\lambda| \leqq \lambda_{0}, \lambda$ real and $m \geqq m\left(\lambda_{0}\right) A_{1}$ and $A_{2}$ are satisfied ${ }^{2}$, and even by taking $m$ large enough one can take $Q_{1}$ as large as we want.

We now want to show that one can bound the norms of the $\hat{g}$ 's functions by norms of the initial functions $g$.

First suppose that $\operatorname{supp} g \subset \Delta$, and that $\Delta \cap \Delta_{\alpha}=\emptyset$. Let $\eta_{\Delta}$ be a $C_{0}^{\infty}$ function such that $\eta_{\Delta}(x)=1$ if $x \in \Delta$ and such that $\operatorname{dist}\left(\operatorname{supp} \eta_{\Delta}, \Delta_{\alpha}\right)>\frac{1}{2}$ (if $\Delta \cap \Delta_{\alpha}=\emptyset$ ). We have

$$
\left\|\hat{g}^{\alpha}\right\|_{L^{2}}^{2}=\int d p d r d t d q \frac{\tilde{g}(p) \tilde{\eta}_{\Delta}(-p+r) \tilde{X}_{\Delta_{\alpha}}(-r+t) \tilde{\eta}_{\Delta}(-t+q) \tilde{\bar{g}}(-q)}{\left(r^{2}+m^{2}\right)^{1 / 4}\left(t^{2}+m^{2}\right)^{1 / 4}}
$$

defining $D:=\left(P^{2}+m^{2}\right)$, then:

$$
\begin{aligned}
\left\|\hat{g}^{\alpha}\right\|_{L^{2}}^{2} & =\left\|g \eta_{\Delta} D^{-1 / 4} \chi_{\Delta_{\alpha}} D^{-1 / 4} \eta_{\Delta} g\right\|_{1} \\
& =\left\|g D^{-1 / 4} D^{1 / 4} \eta_{\Delta} D^{-1 / 4} \chi_{\Delta_{\alpha}} D^{-1 / 4} \eta_{\Delta} D^{1 / 4} D^{-1 / 4} g\right\|_{1} \\
& \leqq\|g\|_{-1 / 2}^{2}\left\|D^{1 / 2} \eta_{\Delta} D^{-1 / 4} \chi_{\Delta_{\alpha}} D^{-1 / 4} \eta_{\Delta} D^{1 / 2} \eta_{\Delta} D^{-1 / 4} \chi_{\Delta_{\alpha}} D^{-1 / 4} \eta_{\Delta}\right\|_{1}
\end{aligned}
$$

where

$$
\|g\|_{-1 / 2}=\left(\int|\tilde{g}(p)|^{2}\left(p^{2}+1\right)^{-1 / 2} d p\right)^{1 / 2} \geqq\left\|D^{-1 / 4} g\right\|_{L^{2}} .
$$

Using then the Theorem 2.2 of Seiler and Simon [14]:

$$
\begin{aligned}
\left\|\hat{g}^{\alpha}\right\|_{L^{2}}^{2} & \leqq\|g\|_{-1 / 2}^{2} e^{-2 Q_{7} d\left(\Delta, \Delta_{\alpha}\right)}\left\|D^{1 / 2} \eta_{\Delta} D^{-1 / 4} \chi_{\Delta_{\alpha}} D^{-1 / 8}\right\|_{2}^{2} \\
& \leqq O(l) e^{-Q_{7} d\left(\Delta, \Delta_{\alpha}\right)}\|g\|_{-1 / 2}^{2}
\end{aligned}
$$

where $Q_{7}$ is as big as we want if $m$ is big enough, and $d\left(\Delta, \Delta_{\alpha}\right)=\sup \left(1, \operatorname{dist}\left(\Delta, \Delta_{\alpha}\right)\right)$.

2 Remark that the theory depends only of the ratio $\lambda / m$ 
Then if $\Delta \cap \Delta_{\alpha} \neq \emptyset$, we use

$$
\left\|\hat{g}^{\alpha}\right\|_{L}^{2}{ }^{2}=\int\left|\left(D^{-1 / 4} g\right)(x)\right|^{2} \chi_{\Delta_{\alpha}}(x) d x \leqq\|g\|_{-1 / 2}^{2} .
$$

We have thus obtained that:

$$
\left\|\hat{g}^{\alpha}\right\|_{L^{2}} \leqq\|g\|_{-1 / 2}^{2} O(1) e^{-Q_{7} d\left(\Delta, \Delta_{\alpha}\right)} .
$$

Using then the fact that $\sum_{\Delta_{\alpha}} e^{-d\left(\Delta, \Delta_{\alpha}\right)} \leqq O(1)$ we obtain for the original Schwinger functions bounds in $\|g\|_{-1 / 2}$ and $\|h\|_{-1 / 2}$, and the exponential decrease between the support of the $\hat{g}$ and $\hat{h}$ give exponential decreases between the support of the $g$ and $h$ using the exponential decrease:

$$
e^{-\left(Q_{7}-1\right) d\left(\Delta, \Delta_{\alpha}\right)} \text {. }
$$

Then as a consequence of $A_{1}$ and $A_{2}$ we get

Theorem 1. Let $\lambda_{0}>0$ be given.

Uniformly in $s$, and $\lambda,|\lambda|<\lambda_{0}$, these exists $m$ large enough such that:

$$
\lim _{\Lambda \rightarrow \infty} \frac{S_{(s), \Lambda}(f ; g ; h)}{Z_{(s), \Lambda}}
$$

exists and is bounded by

$$
O(1)^{n} O(1)^{N} \prod_{i=1}^{n}\left\|f_{i}\right\|_{-1} \prod_{k=1}^{N}\left\|g_{k}\right\|_{-1 / 2}\left\|h_{k}\right\|_{-1 / 2} \prod_{\Delta}(n(\Delta) !)^{1 / 2} .
$$

Moreover, there is an exponential clustering which is as big as we want if $m$ is taken large enough.

Finally under the same conditions as for Theorem 1 , and $S_{A}$ being defined as in (I.1), one has:

Theorem 2. The infinite volume limits $\lim _{\Lambda \rightarrow \infty} Z_{A}^{-1} S_{\Lambda}(f ; g ; h)$ exists and satisfy all the Osterwalder-Schrader axioms, including an exponential clustering.

As an obvious consequence:

Corollary 1. There exists a 2 dimensional Yukawa relativistic theory satisfying the Wightman axioms and possessing a mass gap.

Theorem 2 follows from Theorem 1. Indeed we proceed as in [9]. We define new Schwinger functions $S_{A ; Y}$, for $Y$ a big square union of lattice squares and containing $\Lambda$, by:

$$
S_{A ; Y}:=S_{(s), A} \text { for } s_{b}=1 \text { if } b \in B_{Y} \text { and } s_{b}=0 \text { otherwise. }
$$

$B_{Y}$ is the set of lattice lines strictly contained in $Y$. Then by the equivalent of Proposition IV.1.3 of [9]:

$$
\lim _{Y \rightarrow \infty} S_{\Lambda ; Y}=S_{A} .
$$


On the other hand from Theorem 1, it follows that

$$
\lim _{\Lambda \rightarrow \infty} \lim _{Y \rightarrow \infty} \frac{S_{\Lambda ; Y}}{Z_{\Lambda ; Y}}
$$

exists; this proves the existence part of Theorem 2. Now since this limit is also the limit of the theory defined by Seiler [13] and McBryan [10], it is obvious that all the Osterwalder Schrader axioms are satisfied.

It remains thus to prove $A_{1}$ and $A_{2}$. The proof of $A_{1}$ results from Seiler [13]. In fact $\left.C_{(s)}(x, y)\right|_{\Delta}$ and $\left.K_{(s)}(x, y)\right|_{\Delta}$ are proportional to $C(x, y)$ and $K(x, y), x, y \in \Delta$. Thus the proof of $Z_{(s), \Delta}>0$ reduces itself to the Seiler's proof of $Z \neq 0$ for the volume $\Lambda=\Delta$, i.e. to have $\left\|\left.K\right|_{\Delta}\right\|_{4}^{4}<1$. This condition is obtained by taking $\lambda / m$ small enough.

The remaining of the article is devoted to the proof of $A_{2}$.

\section{The Combinatoric of the Cluster Expansion}

We give now the explicit form of $H$ which is a function of a parameter $m_{1}$, and of $M$ which is a function of another parameter $m_{2}$ :

Definition. Let $m_{1}>0$ and $m_{2}>0$ :

$$
H_{m_{1}}\left(s ; \Delta_{1}, \Delta_{2}, \Delta_{3}\right)=\sum_{\gamma \subset Z^{2^{*}}} \prod_{b \in \gamma} s_{b} \prod_{b \notin \gamma}\left(1-s_{b}\right) \frac{\bar{C}_{m_{1}}^{\gamma}\left(\Delta_{1} ; \Delta_{3}\right)}{\bar{C}_{m_{1}}\left(\Delta_{1} ; \Delta_{3}\right)} \frac{\bar{C}_{m_{1}}^{\gamma}\left(\Delta_{3} ; \Delta_{2}\right)}{\bar{C}_{m_{1}}\left(\Delta_{3} ; \Delta_{2}\right)}
$$

and

$$
\begin{aligned}
M_{m_{2}}\left(\Delta_{1}, \Delta_{2}, \Delta_{3}\right) & =E_{m_{2}}\left(\Delta_{1}, \Delta_{2}, \Delta_{3}\right)\left[\sum_{\alpha} E_{m_{2}}\left(\Delta_{1}, \Delta_{2}, \Delta_{\alpha}\right)\right]^{-1} \\
E_{m_{2}}\left(\Delta_{1}, \Delta_{2}, \Delta_{3}\right) & =e^{-m_{2}\left[d\left(\Delta_{1}, \Delta_{3}\right)+d\left(\Delta_{3}, \Delta_{2}\right)\right]} \\
C_{m_{1}}^{\gamma}(x, y) & =\int e^{-m_{1}^{2} T} \int \prod_{b \in \gamma^{c}} J_{b}^{T}(z) d z_{x y}^{T} d T
\end{aligned}
$$

where $d z_{x y}^{T}$ is the Wiener density for the paths in $R^{2}$ and

$$
\begin{aligned}
J_{b}^{T}(z) & = \begin{cases}0 & \text { if } z(\tau) \in b \quad 0 \leqq \tau \leqq T \\
1 & \text { otherwise }\end{cases} \\
\bar{C}_{m_{1}}^{\gamma}\left(\Delta_{\alpha}, \Delta_{\beta}\right) & =\int C_{m_{1}}^{\gamma}(x, y) \chi_{\Delta_{\alpha}}(x) \chi_{\Delta_{\beta}}(y) d x d y, \quad \bar{C}_{m_{1}}=\bar{C}_{m_{1}}^{Z^{*}}
\end{aligned}
$$

Proof of Lemma I.1. It is obvious. In particular $H$ being a convex sum of non negative quantities smaller than one, we have $0 \leqq H \leqq 1$. If $s \equiv 1$ then in formula (II.1) the only contribution to the sum over $\gamma$ is for $\gamma=Z^{2 *}$ and so $H \equiv 1$.

Finally remark that if $\bar{C}_{m_{1}}^{\gamma}(. ;)=.\left.\sum_{k} \bar{C}_{m_{1}}^{\gamma}(. ;)\right|_{.X_{k}}$ then $\bar{C}_{m_{1}}^{\gamma}\left(\Delta_{1} ; \Delta_{3}\right) \bar{C}_{m_{1}}^{\gamma}\left(\Delta_{3} ; \Delta_{2}\right)$ is equal to zero if $\Delta_{1}, \Delta_{2}$, and $\Delta_{3}$ don't belong to the same $X_{k}$. This finishes the proof.

We now reduce $A_{2}$ to a proposition whose proof is given in Chapter III. This is obtained through a lemma showing that $H_{m_{1}} E_{m_{2}}$ has essentially the same combinatoric properties as Dirichlet covariances, see [8]. Thus let us consider $\partial^{T} \tilde{S}_{(s)}$. 
The $d / d s$ derivations acting on $C(s)$ or $K(s)$ are localized:

$$
\frac{d}{d s} C(s)=\sum_{\Delta, \Delta^{\prime}} \sum_{\alpha} \frac{d}{d s} H\left(s ; \Delta, \Delta^{\prime}, \Delta_{\alpha}\right) M\left(\Delta, \Delta^{\prime}, \Delta_{\alpha}\right) \chi_{\Delta} C \chi_{\Delta^{\prime}}
$$

with obvious notations

$$
\frac{d}{d s} K(s)=\sum_{\Delta, \Delta^{\prime}, \Delta^{\prime \prime}} \frac{d}{d s} H\left(s ; \Delta, \Delta^{\prime}, \Delta^{\prime \prime}\right) \chi_{\Delta} K_{\Delta^{\prime \prime}} \chi_{\Delta^{\prime}} .
$$

We then can write (see [8]):

$$
\partial^{\Gamma} \tilde{S}_{(s)}=\sum_{\substack{\pi \in \mathscr{P}(\Gamma) \\ \pi=\left\{\gamma_{1}, \ldots, \gamma_{L}\right\}}} \sum_{\substack{\Delta_{1}, \Delta_{1}^{\prime}, \Delta_{1}^{\prime \prime} \\ \vdots \\ \Delta_{L}, \Delta_{L}^{\prime}, \Delta_{L}^{\prime \prime}}} \partial^{\gamma_{j}} H\left(s ; \Delta_{j}, \Delta_{j}^{\prime}, \Delta_{j}^{\prime \prime}\right) \sum_{R} \int R d \mu
$$

where $R$ contains $L, K$, or $C$. $M$ localized in $\left(\Delta_{i}, \Delta_{i}^{\prime}, \Delta_{i}^{\prime \prime}\right)$ and $\mathscr{P}(\Gamma)$ is the set of all the partitions of $\Gamma$.

Now the following lemma summarizes the cluster expansion estimates:

Lemma II.1. Let $b_{j}$ be an arbitrary element of $\gamma_{j}$, for $j=1, \ldots, L$ and let $Q_{4}$ and $Q_{3}$ be any positive constants, then there exist a positive constant $Q_{2}$ and $m_{1}$ and $m_{2}$, $m_{2}>m_{1}$ such that:

$$
\begin{aligned}
& \sum_{\substack{\pi \in \mathscr{P}_{(I)}(\Gamma) \\
\pi=\left\{\gamma_{1}, \ldots, \gamma_{L}\right\}}} \sum_{\substack{\Delta_{1}, \Delta_{1}^{\prime}, \Delta_{1}^{\prime \prime} \\
\vdots \\
\Delta_{L}, \Delta_{L}^{\prime}, \Delta_{L}^{\prime \prime}}} \prod_{j=1}^{L}\left\{\partial^{\gamma_{j}} H\left(s ; \Delta_{j}, \Delta_{j}^{\prime}, \Delta_{j}^{\prime \prime}\right) E\left(\Delta_{j}, \Delta_{j}^{\prime}, \Delta_{j}^{\prime \prime}\right) e^{\left.-Q_{2} e^{Q_{3}\left[d\left(b_{j}, \Delta_{j}\right)+d\left(b_{j}, \Delta_{j}^{\prime}\right)\right]}\right\}}\right. \\
& \leqq \\
& \leqq e^{-Q_{4}|\Gamma|} .
\end{aligned}
$$

Proof. Let $\gamma \subset \gamma_{0}$, we define:

$$
\partial^{\gamma} \bar{C}_{m_{1}}^{\gamma_{0}}\left(\Delta, \Delta^{\prime}\right)=\iint e^{-m_{1}^{2} T} \int \prod_{b \in \gamma}\left(1-J_{b}^{T}(z)\right) \prod_{\gamma_{0}^{c}} J_{b}^{T}(z) d z_{x y}^{T} \chi_{\Delta}(x) \chi_{\Delta^{\prime}}(y) d x d y .
$$

Then:

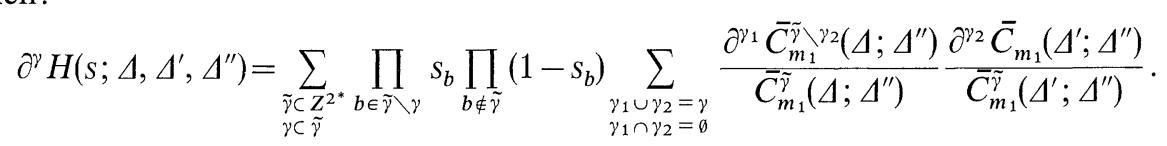

Using

$$
\begin{aligned}
& e^{-m_{2} d\left(\Delta, \Delta^{\prime \prime}\right)}\left[\bar{C}_{m_{1}}\left(\Delta ; \Delta^{\prime \prime}\right)\right]^{-1} \leqq O(1) e^{-\left(m_{2}-m_{1}-1\right) d\left(\Delta, \Delta^{\prime \prime}\right)} \\
& \sum_{\Delta^{\prime \prime}} \exp \left\{-d\left(\Delta, \Delta^{\prime \prime}\right)-d\left(\Delta^{\prime}, \Delta^{\prime \prime}\right)\right\} \leqq O(1)
\end{aligned}
$$

and $\partial^{\gamma} \overline{C^{\gamma}} \leqq \partial^{\gamma} \bar{C}$ we obtain:

$$
\begin{aligned}
\sum_{\Delta^{\prime \prime}} \partial^{\gamma} H\left(s ; \Delta, \Delta^{\prime}, \Delta^{\prime \prime}\right) E\left(\Delta, \Delta^{\prime}, \Delta^{\prime \prime}\right) \leqq & O(1) \sum_{\substack{\gamma_{1} \cup \gamma_{2}=\gamma \\
\sup ^{\prime \prime}}} \partial^{\gamma_{1}} \bar{C}_{m_{1}}\left(\Delta ; \Delta^{\prime \prime}\right) \\
& \cdot e^{-\left(m_{2}-m_{1}-2\right)\left[d\left(\Delta, \Delta^{\prime \prime}\right)+d\left(\Delta^{\prime}, \Delta^{\prime \prime}\right)\right]} \partial^{\gamma_{2}} \bar{C}_{m_{1}}\left(\Delta^{\prime} ; \Delta^{\prime \prime}\right) .
\end{aligned}
$$

In formula (II.3), for any $\pi \in \mathscr{P}(\Gamma), \pi=\left\{\gamma_{1}, \ldots, \gamma_{L}\right\}$ let $b_{i}$ be any element of $\gamma_{i}$ then:

$$
\sum_{\left\{\Delta_{i}, \Delta_{i}^{\prime}\right\}} \prod_{j=1}^{L} e^{-\left[d\left(b_{j}, \Delta_{j}\right)+d\left(b_{j}, \Delta_{j}^{\prime}\right)\right]} \leqq O(1)^{L} .
$$


To finish the proof it is then sufficient to prove

$$
\begin{aligned}
& \sum_{\substack{\left.\pi \in \mathscr{P}(\Gamma), \bar{y}^{2}\right\} \\
\pi=\left\{\bar{\gamma}_{1}, \ldots, \bar{\gamma}_{L}\right\}}} \sum_{\substack{\gamma_{i} \cup \gamma_{i}^{\prime}=\bar{\gamma}_{2} \\
\gamma_{2} \cap \gamma_{2}^{\prime}=\emptyset}} \sup _{\Delta_{l}, \Delta_{l}^{\prime}, \Delta_{\alpha_{i}}} \prod_{i=1}^{L}\left\{\partial^{\gamma_{i}} \bar{C}_{m_{1}}\left(\Delta_{i}, \Delta_{\alpha_{l}}\right) \partial^{\gamma_{i}^{\prime}} \bar{C}_{m_{1}}\left(\Delta_{i}^{\prime}, \Delta_{\alpha_{l}}\right)\right. \\
& \left.\cdot e^{-\left(m_{2}-m_{1}-2\right)\left[d\left(\Delta_{l}, \Delta_{\alpha_{l}}\right)+d\left(\Delta_{l}^{\prime}, \Delta_{\alpha_{l}}\right)\right]} e^{\left(Q_{3}+1\right)\left[d\left(b_{l}, \Delta_{\imath}\right)+d\left(b_{l}, \Delta_{l}^{\prime}\right)\right]} O(1) e^{-Q_{2}}\right\} \leqq e^{-Q_{0}|\Gamma|}
\end{aligned}
$$

where $b_{i}$ is any element of $\bar{\gamma}_{i}$. Suppose that $b_{i} \in \gamma_{i}$, we have

$$
d\left(b_{i}, \Delta_{i}^{\prime}\right) \leqq d\left(\Delta_{i}, \Delta_{i}^{\prime}\right)+d\left(b_{i}, \Delta_{i}\right)+2 \leqq d\left(\Delta_{i}, \Delta_{\alpha_{i}}\right)+d\left(\Delta_{\alpha_{i}}, \Delta_{i}^{\prime}\right)+d\left(b_{i}, \Delta_{i}\right)+4 .
$$

If $\gamma_{i}=\emptyset\left(\right.$ or $\gamma_{i}^{\prime}=\emptyset$ ) then we use: $\bar{C}_{m_{1}}\left(\Delta_{i} . \Delta_{\alpha_{1}}\right) \leqq O(1)$ and we can choose the parameters such that:

$$
O(1) e^{-Q_{2 / 2}} e^{2\left(Q_{3}+1\right)} e^{-\left(m_{2}-m_{1}-Q_{3}-3\right) d\left(\Delta_{i}, \Delta_{\alpha_{1}}\right)} \leqq 1
$$

so that in the remaining of the proof we can forget about the empty $\gamma_{i}$. We symmetrize: let now $b_{i}$ (resp. $b_{i}^{\prime}$ ) be an arbitrary element of $\gamma_{i}\left(\right.$ resp. $\left.\gamma_{i}^{\prime}\right)$ then for $\gamma_{i} \neq \emptyset$ we define:

$$
\begin{gathered}
f\left(\gamma_{i}\right)=\sup _{\tilde{\Delta}_{l}, \tilde{\Delta}_{l}} \partial^{\gamma_{l}} \bar{C}_{m_{1}}\left(\tilde{\tilde{\Delta}}_{i}, \tilde{\Delta}_{i}\right) e^{-\left(m_{2}-m_{1}-Q_{3}-3\right) d\left(\tilde{\Delta}_{l}, \tilde{\Delta}_{l}\right)} e^{2\left(Q_{3}+1\right) d\left(b_{l}, \Delta_{l}\right)} \\
\cdot e^{2\left(Q_{3}+1\right)} O(1) e^{-Q_{2 / 2}} e^{d\left(b_{l}, \tilde{\Delta}_{l}\right)+d\left(\tilde{\Delta}_{l}, \tilde{\tilde{u}}_{l}\right)}
\end{gathered}
$$

and for $\gamma_{i}=\emptyset, f(\emptyset)=1$.

To prove (II.3) it is sufficient to prove that:

$$
\sum_{\substack{\pi \in \mathscr{P}(\Gamma) \\ \pi=\left\{\bar{\gamma}_{i}, \ldots, \bar{\gamma}_{L}\right)}} \sum_{\substack{\gamma_{1} \cup \gamma_{2}^{\prime}=\bar{\gamma}_{i} \\ \gamma_{i} \cap \gamma_{i}^{\prime}=\emptyset}} \prod_{i} O(1) e^{-d\left(b_{i}, b_{i}^{\prime}\right)} f\left(\gamma_{i}\right) f\left(\gamma_{i}^{\prime}\right) \leqq e^{-Q_{4}|\Gamma|} .
$$

We assert then that the 1.h.s. in (II.4) is smaller than ${ }^{3}$ :

$$
\sum_{\substack{\pi \in \mathscr{P}(\Gamma) \\ \pi=\left(\tilde{\gamma}_{i} \ldots \tilde{\gamma}_{p}\right)}} O(1)^{p} 2^{p} \prod_{i=1}^{p} f\left(\tilde{\gamma}_{i}\right)
$$

the proof that this is smaller than $e^{-Q_{4}|\Gamma|}$ for a correct choice of $Q_{2}, m_{1}\left(m_{2}>m_{1}\right)$ is given in the references [8] and [15] and is one of the main combinatoric tool of the cluster expansion. This finishes the proof of the lemma.

With this lemma the proof of $A_{2}$ is reduced to the proof of:

Proposition II.1. For given $Q_{2}, \lambda_{0}$, and $m_{2}$ (large) there exists $Q_{3}$ and $Q_{6}$ (independant of the parameters) such that for $m$ large enough and $|\lambda|<\lambda_{0}$ (see formula II.2):

$$
\begin{aligned}
& \sup _{L} \sup _{\substack{b_{1} \in \Gamma \\
j=1, \ldots, L}} \sup _{\substack{\Delta_{1}, \Delta_{1}^{\prime}, \Delta_{1}^{\prime \prime} \\
\vdots \\
\Delta_{L}, \Delta_{L}^{\prime}, \dot{\Delta}_{L}^{\prime \prime}}} \prod_{i=1}^{L}\left\{e^{-Q_{3}\left[d\left(\Delta_{j}, b_{j}\right)+d\left(b_{j}, \Delta_{j}^{\prime}\right)\right]} e^{Q_{2}}\right\} \\
& \prod_{\text {derived } k} e^{m_{2}\left[d\left(\Delta_{j}, \Delta_{j}^{\prime \prime}\right)+d\left(\Delta_{j}^{\prime}, \Delta_{j}^{\prime}\right)\right]} \prod_{\text {derived } C} e^{2 m_{2} d\left(\Delta_{j}, \Delta_{j}^{\prime}\right)} \sum_{R}\left|\int R d \mu\right| \\
& \leqq O(1)^{n} O(1)^{N} \prod_{\Delta}(n(\Delta) !)^{1 / 2} e^{Q_{6}|\Gamma|} \prod_{i=1}\left\|f_{i}\right\|_{-1} \prod_{j=1}^{N}\left\|\hat{g}_{j}\right\|_{L^{2}}\left\|\hat{h}_{j}\right\|_{L_{2}} .
\end{aligned}
$$

Remark. We have used that:

$$
\begin{aligned}
E^{-1}\left(\Delta_{j}, \Delta_{j}^{\prime}, \Delta_{j}^{\prime \prime}\right) & =\exp \left\{m_{2}\left[d\left(\Delta_{j}, \Delta_{j}^{\prime \prime}\right)+d\left(\Delta_{j}^{\prime}, \Delta_{j}^{\prime \prime}\right)\right]\right\} \\
\left(E^{-1} M\right)\left(\Delta_{j}, \Delta_{j}^{\prime}, \Delta_{j}^{\prime \prime}\right) & \leqq \exp \left\{2 m_{2} d\left(\Delta_{j}, \Delta_{j}^{\prime}\right)\right\}
\end{aligned}
$$

3 It has been pointed out by Lon Rosen that this inequality in a preliminary version and the corresponding formula in [9] are incorrect 
and $R$ is defined by what we obtain after performing the derivations up to the factors $\partial^{\gamma} H$ and $M$.

Also in the formula above $O(1)$ in $O(1)^{n}$ depends on $Q_{2}$.

Then $A_{2}$ follows from the fact that one can take $Q_{4}$ as large as we want by choosing $m_{2}$ (and thus $m$ ) large enough and define $Q_{1}=Q_{4}-Q_{6}$.

\section{The Cluster Expansion: Proof of Proposition II.1}

First let us see what we obtain when we do a derivation $d / d s_{b}, b \in \Gamma$ on a Schwinger function $\tilde{S}(\omega)$

$$
\begin{aligned}
& \tilde{S}(\omega)=\iint \operatorname{det}_{j k} S_{F}\left(x_{j}, y_{k} ; \phi\right) \prod \phi\left(t_{i}\right) \operatorname{det}_{\mathrm{ren}}(1+K) \\
& \omega\left(x_{1} \ldots ; y_{1}, \ldots ; t_{1}, \ldots\right) d \mu \prod_{j} d x_{j} d y_{j} \prod_{i} d t_{i}
\end{aligned}
$$

where $S_{F}(x, y ; \phi)$ stands for the kernel of $(1+K)^{-1}$, and $\omega$ is some function with each argument localized in some unit square of the lattice cover. Also for simplicity since we look at some algebraic aspects we omit any reference to $\lambda, \Gamma$ of $\Lambda$.

Acting on an expression of the form $\int R d \mu$ where $R$ and $d \mu$ depend on $s$, the derivation $d / d s$ produces two categories of terms:

$$
\frac{d}{d s} \int R d \mu=\int \frac{d R}{d s} d \mu+\frac{1}{2} \int \frac{d C}{d s}(x, y)\left(\frac{\partial^{2}}{\partial \phi(x) \partial \phi(y)} R\right) d x d y d \mu .
$$

$R$ being of the form of the integrand in (III.1) one sees that one has essentially to know the effect of $d / d s$ or $\partial / \partial \phi$ on $S_{F}(x, y ; \phi)$ or on $\operatorname{det}_{\text {ren }}(1+K)$.

One gets:

$$
\begin{aligned}
& \frac{d}{d s} \operatorname{det}_{\mathrm{ren}}(1+K) \\
& =\operatorname{det}_{\mathrm{ren}}(1+K)\left\{\sum_{\alpha, \beta} d x d y \chi_{\Delta_{\alpha}}(x) \chi_{\Delta_{\beta}}(y)\right. \\
& \quad \cdot\left[\sum_{\gamma, \delta} \int d w d z \chi_{\Delta_{\gamma}}(z) \chi_{\Delta_{\delta}}(w) S_{F}(y, z ; \phi) K(z, w) K(w, x) \frac{d K}{d s}(x, y)\right. \\
& \left.\left.-\frac{1}{2}: \phi(x) \frac{d}{d s} b_{\mathrm{reg}}(x, y) \phi(y):\right]\right\} \\
& =: \operatorname{det}_{\mathrm{ren}}(1+K)\left\{\operatorname{Tr} S_{F} A_{2}+A_{3}\right\} \\
& \begin{aligned}
\frac{d}{d s} S_{F}(x, y ; \phi)= & \sum_{\alpha, \beta}\left\{-\int d z \frac{d K}{d s}(x, z) S_{F}(z, y ; \phi) \chi_{\Delta_{\alpha}}(x) \chi_{\Delta_{\beta}}(z)\right. \\
& +\int d z d w \chi_{\Delta_{\alpha}}(z) \chi_{\Delta_{\beta}}(w) K(x, z) \frac{d K}{d s}(z, w) S_{F}(w, y ; \phi) \\
& -\sum_{\gamma, \delta} \int d z d w d v d u \chi_{\Delta_{\alpha}}(v) \chi_{\Delta_{\beta}}(u) \chi_{\Delta_{\gamma}}(z) \chi_{\Delta_{\delta}}(w) \\
& \left.\cdot S_{F}(x, z ; \phi) K(z, w) K(w, v) \frac{d K}{d s}(v, u) S_{F}(u, y ; \phi)\right\} \\
= & : A_{1} S_{F}-S_{F} A_{2} S_{F} .
\end{aligned}
\end{aligned}
$$


The formula of derivations for $\partial / \partial \phi(z)$ are the same with $\frac{\mathrm{K}}{d s}$ replaced by

$$
\begin{aligned}
& \frac{\partial K(x, y)}{\partial \phi(z)}=\sum_{\Delta, \Delta^{\prime}, \Delta^{\prime \prime}} \lambda H\left(s ; \Delta, \Delta^{\prime}, \Delta^{\prime \prime}\right)\left[\left(P^{2}+m^{2}\right)^{-1 / 4} \frac{P+m}{\left(P^{2}+m^{2}\right)^{1 / 2}}\right](x, z) \\
& \chi_{\Delta^{\prime \prime}}(z)\left(P^{2}+m^{2}\right)^{-1 / 4}(z, y) \chi_{\Delta}(x) \chi_{\Delta^{\prime}}(y) .
\end{aligned}
$$

We note $\partial / \partial \phi \operatorname{det}_{\mathrm{ren}}(1+K)=\operatorname{det}_{\mathrm{ren}}(1+K)\left\{\operatorname{Tr} S_{F} A_{2}^{\prime}+A_{3}^{\prime}\right\}$

$$
\frac{\partial}{\partial \phi} S_{F}=A_{1}^{\prime} S_{F}-S_{F} A_{2}^{\prime} S_{F}
$$

By their definition the $A_{i}$ or $A_{i}^{\prime}$ are completely localized expressions. In each of these terms between any two localization squares there is always a chain of "propagators":

$$
\left(P^{2}+m^{2}\right)^{-1 / 4}(x, y) \quad \text { or }\left[\left(P^{2}+m^{2}\right)^{-1 / 4} \frac{P+m}{\left(P^{2}+m^{2}\right)^{1 / 2}}\right](x, y) .
$$

Each boson propagator, each boson field and each propagator is localized.

The $A_{i}$ and $A_{i}^{\prime}$ are polynomials in the boson field of degree 3 at most, and between any two such fields there is always a chain of "propagators". Each $A_{i}$ or $A_{i}^{\prime}$ has at most $3 K$. Finally, acting on $S_{F}$ a derivation generates an expression or order 2 in $S_{F}$, and acting on $\operatorname{det}_{\text {ren }}(1+K)$ an expression of degree 1 in $S_{F}$.

Before estimating the number of terms produced by derivation we have first to reorder them in view of preserving the antisymmetric structure since it is essential for the volume dependance estimate $([13,10])$. Therefore after each derivation we perform the following operation:

- when the derivation acts on $\operatorname{det}_{i k} S_{F} \operatorname{det}_{\text {ren }}(1+K)$ put together the terms which increase the degree in $S_{F}$. They form a new determinant of one order higher (this can be checked easily).

- other terms are left unchanged.

Now as a first step in proving Proposition II.1 we bound the number of terms produced by the derivations. To do this we use the technique of the combinatoric factors (see [7]).

To take account of the sum over the localization squares we need exponential localization factors of the type $e^{O(1) d\left(\Delta, \Delta^{\prime}\right)}$ or $e^{O(1) d(b, \Delta)}$ where $\Delta$ and $\Delta^{\prime}$ are two localization squares in an $A_{i}$ or $A_{i}^{\prime}$ generated by a derivation relatively to $s_{b}$.

Two localizations squares in an $A_{i}$ or $A_{i}^{\prime}$ are linked by a chain of "propagators".

There is at most 6 "propagators" by chain. We thus distribute the localization factors to the "propagators" using:

$$
\begin{aligned}
& d\left(\Delta, \Delta^{\prime}\right) \leqq d\left(\Delta, \Delta_{1}\right)+\ldots+d\left(\Delta_{i}, \Delta^{\prime}\right)+10 \quad(i \leqq 5) \\
& d(b, \Delta) \leqq d\left(b, \Delta_{1}\right)+\ldots+d\left(\Delta_{i}, \Delta\right)+12 \quad(i \leqq 6) .
\end{aligned}
$$

Let $L$ be the number of derived $K$ or $C$. We take account of these factors by the following combinatoric factors:

$$
O(1)^{L} \prod_{\text {derivations }} e^{O(1)\left[d\left(b, \Delta_{1}\right)+d\left(b, \Delta_{2}\right)\right]} \prod_{\text {"propagators" }} e^{O(1) d\left(\Delta, \Delta^{\prime}\right)}
$$


where product over the derivations means product over the derived $K$ or $C$, $\Delta_{1}$, and $\Delta_{2}$ being the localizations squares of $K(x, y)$ or $C(x, y)$ and $b$ is one of the bond relatively to which $K$ or $C$ is derived.

We will not list these factors in the following.

Because of Lemma II.1 we don't need to take account of how many times, and relatively to what set of bonds a $K$ or $C$ is derived i.e. we count only the derivations acting on a non derived $K$ or $C$. In a given term we attribute a factor 2 to each $K$ or $C$ to decide whether it is derived or not. This gives a $2^{O(1) L}$ since the number of $K$ or $C$ is bounded by $O(1) L$.

Also for each derived $A_{i}, A_{i}^{\prime}$ we fix each localization square $\Delta$ using a combinatoric factor $O(1) e^{d(b, \Delta)}$.

We are thus ready to compute the number of terms generated by the derivations. Giving a factor 2 to each derivation we separate its effect according to the following cases.

a) $\frac{d}{d s}$ acts on everything except the measure $d \mu$.

B) $\frac{d}{d s}$ acts on $d \mu$.

We first compute the combinatoric factors for the case $\alpha$. With a factor $O(1)$ given to the derivation, we divide case $\alpha$ in several subcases:

$\left.\alpha_{1}\right) \frac{d}{d s}$ derives $S_{F}$ and we select $A_{1} S_{F}$.

$\alpha_{2}$ ) We consider the sum of terms of higher degree in $S_{F}$ which form a determinant of higher order.

$\left.\alpha_{3}\right) \frac{d}{d s}$ derives $\operatorname{det}_{\text {ren }}(1+K)$ and we select $A_{3}$.

$\left.\alpha_{4}\right) \frac{d}{d s}$ derives a $K$ or $C$ created by a previous derivation (i.e. a fermion propagator in $A_{i}$ or $A_{i}^{\prime}$ ).

Let us consider the combinatoric for each case separately (excluding the localization factors).

Case $\alpha_{4}$ : the combinatoric factor is 1 since the derived propagators have already been chosen.

Case $\alpha_{3}$ : the combinatoric factor is 1 since there is only one term.

Case $\alpha_{2}$ : the combinatoric factor is 1 since there is only one term.

Case $\alpha_{1}$ : it is the case: $S_{F}(x, y ; \phi) \rightarrow\left(A_{1} S_{F}\right)(x, y)$.

Let $i$ in $\operatorname{det}_{i k} S_{F}\left(x_{i}, y_{k} ; \phi\right)$ labels the columns. The term given by the determinant in case $\alpha_{1}$ is a sum of determinant each with a column $A_{1} S_{F}$. The combinatoric factors we are looking for control the number of columns (initial or produced by derivations). With a factor 2 we distinguish 2 subcases:

a) the smearing function for the variable $x$ is a $A_{i}$ or $A_{i}^{\prime}$ created by a previous derivation.

b) the smearing function for the variable $x$ is an original $\hat{g}_{i}$ of formula (I.2). 
We consider first the case a). The function $A_{i}\left(\right.$ or $\left.A_{i}^{\prime}\right)$ has been produced by a localized derivation $\frac{d}{d s}$ and thus contains:

$\chi_{\Delta_{1}}(u) \frac{d K}{d s}(u, v) \chi_{\Delta_{2}}(v)$ or has been produced by $\chi_{\Delta_{2}}(z) \frac{\delta}{\partial \phi(z)}$.

The square $\Delta_{2}$ is chosen with a localization factor $\exp \left\{O(1) d\left(b, \Delta_{2}\right)\right\}$.

Let $R_{a, c}(\Delta)$ be the number of times that in a) $\Delta_{2}=\Delta$. In a given term at the end of the expansion, let $n_{c}(\Delta)$ be the sum of the number of times that in $\frac{d K}{d s}(u, v)$, $v$ is localized in $\Delta$ and of the number of times that in $\frac{d C}{d s}(u, v), u$ or $v$ is localized in $\Delta$. Then the number of $A_{i}$ or $A_{i}^{\prime}$ with $\Delta_{2}=\Delta$ is bounded by $n_{c}(\Delta)$. Doing this choice $R_{a, c}$ times we obtain as combinatoric factor:

$$
\prod_{\Delta}\left\{n_{c}(\Delta)\right\}^{R_{a, c}(\Delta)} \leqq \prod_{\Delta} n_{c}(\Delta)^{n_{c}(\Delta)} \prod_{\Delta} R_{a, c}(\Delta)^{R_{a, c}(\Delta)} .
$$

We then "attribute" to each derivation in case a) with $\Delta_{2}=\Delta$, a localization factor $e^{2 d(b, 4)}$, this attribution allows us to use the following lemma:

\section{Lemma III.1.}

$$
\begin{aligned}
& \prod_{\Delta}\left\{n_{c}(\Delta)^{n_{c}(\Delta)} \prod_{b \text { such that } \Delta_{2}=\Delta} e^{-d(b, \Delta)}\right\} \leqq O(1) O(1)^{L} \\
& \prod_{\Delta}\left\{R_{a, c}(\Delta)^{R_{a, c}(\Delta)} \prod_{b \text { such that } \Delta_{2}=\Delta} e^{-d(b, \Delta)}\right\} \leqq O(1) O(1)^{L} .
\end{aligned}
$$

Proof. The first inequality is just Lemma (10.2) of [8]. The second inequality follows also from this lemma if one remarks that: $R_{a, c}(\Delta) \leqq 2 n_{c}(\Delta)$.

The overall combinatoric factor for case a) is therefore $O(1) O(1)^{L}$.

Consider now case b). Let $N_{c}\left(\Delta_{0}\right)$ be the number of functions $\hat{g}_{i}, i=1, \ldots, N$ which have support in $\Delta_{0}$ (it is also in the determinant the number of columns with functions $\hat{g}_{i}$ localized in $\Delta_{0}$. We choose with a localization factor $e^{O(1) d\left(b, \Delta_{0}\right)}$ the square $\Delta_{0}$ support of the function $\hat{g}_{i}$.

Let now $R_{b, c}(\Delta)$ be the number of times that in case b) $\Delta_{0}=\Delta$. We thus get a combinatoric factor:

$$
\prod_{\Delta} N_{c}(\Delta)^{R_{b, c}(\Delta)} .
$$

Attributing [as in case a)] to each derivation a factor $e^{d(b, \Delta)}$, we have at our disposal a factor $e^{-d(b, \Delta)}$ that we used in the following lemma.

\section{Lemma III.2.}

$$
\prod_{\Delta} N_{c}(\Delta)^{R_{b, c}(\Delta)} \prod_{b \text { such that } \Delta_{0}=\Delta} e^{-d(b, \Delta)} \leqq O(1) O(1)^{N} .
$$

Proof. One has (see [8], Lemma 10.2):

$$
\prod_{b \text { such that } \Delta_{0}=\Delta} e^{-d(b, \Delta)} \leqq O(1)^{-O(1) R_{b, c}(\Delta)^{3 / 2}}
$$


so that:

$$
\begin{aligned}
N_{c}(\Delta)^{R_{b, c}(\Delta)} \prod_{b} e^{-d(b, \Delta)} & \leqq e^{R_{b, c}(\Delta) \ln N_{c}(\Delta)-O(1) R_{b, c}(\Delta)^{3 / 2}} \\
& \leqq O(1) e^{O(1)\left(\ln N_{c}(\Delta)\right)^{3}} \leqq O(1) e^{O(1) N_{c}(\Delta)}
\end{aligned}
$$

but now $\prod_{\Delta} \exp \left\{O(1) N_{c}(\Delta)\right\}=O(1)^{N}$, this proves the lemma.

The total combinatoric factor for case $\alpha_{1}$ is thus $O(1) O(1)^{L} O(1)^{N}$.

We now compute the combinatoric factors for case $\beta$. Since in this case each $d / d s$ derivation generates two $\partial / \partial \phi$ derivations we compute the combinatoric factor of $\partial / \partial \phi$ derivations.

With a factor $O(1)$ by $\partial / \partial \phi$ derivation we divide the effect of $\partial / \partial \phi$ in several cases in analogy to case $\alpha$ :

$\left.\alpha_{1}^{\prime}\right) \partial / \partial \phi$ derives $S_{F}$ and we select $A_{1}^{\prime} S_{F}$.

$\alpha_{2}^{\prime}$ ) We consider the sum of therms of higher degree in $S_{F}$ which form a determinant of higher order.

$\left.\alpha_{3}^{\prime}\right) \partial / \partial \phi$ derives $\operatorname{det}_{\text {ren }}(1+K)$ and we select $A_{3}^{\prime}$. $\left.A_{i}^{\prime}\right)$.

$\left.\alpha_{4}^{\prime}\right) \partial / \partial \phi$ derives fields $\phi$ created by previous derivations (i.e. fields in $A_{i}$ or

$\left.\alpha_{5}^{\prime}\right) \partial / \partial \phi$ derives $\prod_{i=1}^{n} \phi\left(f_{i}\right)$ (of formula I.2).

The derivation $\partial / \partial \phi$ is localized in some square $\Delta_{0}$ (already chosen). Let us now consider the combinatoric factor for each case. Cases $\alpha_{1}^{\prime}, \alpha_{2}^{\prime}$, and $\alpha_{3}^{\prime}$ are as above.

Case $\alpha_{4}^{\prime}$ : the field $\phi(z), z \in \Delta_{0}$, which is derived is in some $A_{1}$ or $A_{i}^{\prime}$ produced by $\chi_{\Delta_{1}}(u) \chi_{\Delta_{2}}(v) \frac{d}{d s}$ or by $\chi_{\Delta_{2}}(t) \frac{\partial}{\partial \phi(t)}$.

We choose $\Delta_{2}$ with a factor $e^{O(1) d\left(\Delta_{0}, \Delta_{2}\right)}$. Now all $A_{i}$ or $A_{i}^{\prime}$ generated by derivations localized in $\Delta_{2}$ have at most $3 n_{c}\left(\Delta_{2}\right)$ fields, since there is at most 3 fields in each $A_{i}$ or $A_{i}^{\prime}$. The combinatoric factor is then:

$$
\prod_{\Delta}\left(3 n_{c}(\Delta)\right)^{3 n_{c}(\Delta)}
$$

We deal with this factor as above, see Lemma III.1, this gives a bound: $O(1) O(1)^{L}$. Case $\alpha_{5}^{\prime}$ : let $R\left(\Delta_{0}\right)$ be the number of times that derivations $\partial / \partial \phi$, acting on $\prod_{i=1}^{n} \phi\left(f_{i}\right)$, are localized in $\Delta_{0}$. Each time the number of choices is $n\left(\Delta_{0}\right)$ (remember it is the number of $f_{i}$ with support in $\Delta_{0}$ ). Thus the total combinatoric factor is

$$
\prod_{\Delta} n(\Delta)^{R(\Delta)}
$$

Attributing a factor $e^{d\left(b, \Delta_{0}\right)}$ to each derivation relative to $b$, localized in $\Delta_{0}$, we have by Lemma III.2:

$$
\prod_{\Delta}\left\{n(\Delta)^{R(\Delta)} \prod_{b \text { deriving in } \Delta} e^{-d(b, \Delta)}\right\} \leqq O(1) O(1)^{n} .
$$


Finally we have got that for the cluster expansion, the combinatoric factors are (see Proposition II.1):

$$
O(1) O(1)^{L} O(1)^{n} O(1)^{N} \prod_{\text {derivations }} e^{O(1)\left[d\left(b_{l}, \Delta_{l}\right)+d\left(b_{i}, \Delta_{i}^{\prime}\right)\right]} \prod_{\text {"propagators" }} e^{O(1) d}
$$

where $d$ stands for $d\left(\Delta, \Delta^{\prime}\right), \Delta$ and $\Delta^{\prime}$ being the localization squares of the "propagator". Define $\bar{\lambda}_{0}=\sup \left\{1, \lambda_{0}\right\}$. Now the following lemma is sufficient to prove Proposition II.1:

Lemma III.3. Fix $m_{1}$ and $m_{2}$ as for Lemma II.1 and let $R$ be an element in the expansion of Proposition II.1, then there exist $Q_{3}$ large (depending on $m_{2}$ and $m_{1}$ ), $m$ large enough and $Q_{5}>0$ such that:

$$
\begin{aligned}
& \sup _{b_{i}, i=1, \ldots, L} \sup _{\substack{\Delta_{1}, \Delta_{1}^{\prime}, \Delta_{1}^{\prime \prime} \\
\vdots \\
\Delta_{L}, \Delta_{L}, \Delta_{L}^{\prime \prime}}} \sup _{R}\left\{\prod_{i=1}^{L} e^{-\left(Q_{3}-O(1)\right)\left[d\left(b_{i}, \Delta_{i}\right)+d\left(b_{i}, \Delta_{i}^{\prime}\right)\right]}\right. \\
& \left.\cdot \prod_{\substack{\text { derived } \\
\text { boson propagators }}} e^{O(1) d} \prod_{\text {"propagators" }} e^{O(1) d} O(1)^{L}\left|\int R d \mu\right|\right\} \\
& \leqq\left[\bar{\lambda}_{0}^{3} e^{-Q_{5}}\right]^{L}\left[e^{Q_{5}} O(1)\right]^{n} O(1)^{N} e^{Q_{6}|\Gamma|} \prod_{\Delta}(n(\Delta) !)^{1 / 2} \prod_{i=1}^{n}\left\|f_{i}\right\|_{-1} \prod_{j=1}^{N}\left\|\hat{g}_{j}\right\|_{L^{2}}\left\|\hat{h}_{j}\right\|_{L^{2}} .
\end{aligned}
$$

$Q_{5}$ can be taken as large as we want if $m$ is taken sufficiently large and is independant of $\Gamma$. The $O(1)$ factors in the right hand side and $Q_{6}$ are independant of $m_{1}, m_{2}, m$, and $\Gamma$.

This lemma includes the combinatoric factors of $\sum_{R}$ in Proposition II.1, thus taking $Q_{2}+3 \log \bar{\lambda}_{0} \leqq Q_{5}$ and the supremum over $L$, one has proved this proposition.

Let us now prove Lemma III.3. The integrand $R$ has the general form:

$$
R=\int \operatorname{det}_{i k} S_{F}\left(x_{i}, y_{k} ; \phi\right) w\left(x_{1}, \ldots ; y_{1}, \ldots\right) \operatorname{det}_{\text {ren }}(1+K) d x_{1} \ldots d y_{1} \ldots
$$

where $w\left(x_{1}, \ldots ; y_{1}, \ldots\right)$ is a product or integral of product of $\hat{g}_{i}, \hat{h}_{k}, C, A_{i}, A_{i}^{\prime}$ and $\phi(f)$.

To bound $\left|\int R d \mu\right|$ we use:

Proposition III.1. Let $1 \leqq i, k \leqq N+2 L$, and $M$ (in the definition of $\operatorname{det}_{\mathrm{ren}}(1+K)$ be large enough depending on $\lambda_{0}$ then there exists $Q_{6}>0$ :

$$
\begin{aligned}
& \left|\int \operatorname{det}_{i k} S_{F}\left(x_{i}, y_{k} ; \phi\right) w\left(x_{1}, \ldots ; y_{1}, \ldots\right) \operatorname{det}_{\mathrm{ren}}(1+K) d x_{1} \ldots d y_{1} \ldots d \mu\right| \\
& \leqq\left[\int\|w\|_{L^{2}}^{4} d \mu\right]^{1 / 4} O(1) e^{Q_{6}|\Lambda|} O(1)^{N+2 L} .
\end{aligned}
$$

Proof. Applying twice Schwarz inequality the proposition follows from the work of Seiler and Simon [14]: their proof applies here since they have also localized each $K(x, y)$ in unit squares, the fact of multiplying it by $0 \leqq H\left(\Delta, \Delta, \Delta^{\prime}\right) \leqq 1(x \in \Delta$, $\left.y \in \Delta^{\prime}\right)$ leaves the proof available up to obvious modifications. 
Note: Our preprint version [16] was self contained and in particular included a third proof of the linear lower bound, which differs from those of McBryan [10] and [11] and of Seiler and Simon [14].

It seems also to us that the nice proof of McBryan can also be extended to the $s$-dependant models described here.

Under the conditions of $A_{2},|\Lambda|=|X| \leqq|\Gamma|+1(|X|=$ surface of $X)$. The next step is to bound $\int\|w\|_{L^{2}}^{4} d \mu$.

First we estimate the effect of the functional integration $d \mu$ on $\|w\|^{4}$ by computing the combinatoric factors corresponding to the contractions between the fields $\phi$.

We characterize the fields $\phi$ by squares:

1) For a field $\phi(f)$ of formula (I.1), the "characterizing square" is $\Delta$ if support of $f_{i}$ is in $\Delta$.

2) For a field belonging to some $A_{i}\left(\right.$ resp. $\left.A_{i}^{\prime}\right)$ the characterizing square is $\Delta$ if $A_{i}\left(\right.$ resp. $\left.A_{i}^{\prime}\right)$ has been generated by $\chi_{\Delta}(x) \frac{d}{d s} K(x, \cdot),\left(\right.$ resp. by $\chi_{\Delta}(x) \frac{d}{d s} C(x, \cdot)$ or by $\left.\chi_{\Delta}(y) \frac{d}{d s} C(\cdot, y)\right)$.

The number of fields characterized by $\Delta$ is less than $4 n(\Delta)+12 n_{c}(\Delta)$ (since there is at most 3 fields $\phi$ by $A_{i}$ or $A_{i}^{\prime}$ ).

Attributing a factor $e^{O(1) d\left(\Delta, \Delta^{\prime}\right)}$ to each contraction between a field characterized by $\Delta$ with a field characterized by $\Delta^{\prime}$, we get as a combinatoric factor for the contractions:

$$
\begin{aligned}
& \prod_{\Delta} O(1)^{4 n(\Delta)+12 n_{c}(\Delta)}\left|\left[4 n(\Delta)+12 n_{c}(\Delta)\right] !\right|^{1 / 2} \\
& \leqq O(1)^{n} O(1)^{L} \prod_{\Delta}(4 n(\Delta) !)^{1 / 2} \prod_{\Delta}\left(12 n_{c}(\Delta) !\right)^{1 / 2} .
\end{aligned}
$$

Attributing factors $e^{O(1) d(b, \Delta)}$ to the derivations we treat the last term as before.

Finally the (localization) factors $e^{O(1) d\left(\Delta, \Delta^{\prime}\right)}$ can be decomposed in a product of localization factors by boson propagator and by "propagator".

The total combinatoric factor for the contractions is thus for $\left|\int\|w\|_{L^{2}}^{4} d \mu\right|^{1 / 4}$ :

$$
O(1)^{n} O(1)^{L} \prod_{\Delta}(n(\Delta) !)^{1 / 2} \prod_{\text {derivations }} e^{O(1)\left[d\left(\Delta_{l}, b_{l}\right)+d\left(b_{i}, \Delta_{l}^{\prime}\right)\right]} \prod_{\text {boson propagators }} e^{O(1) d} .
$$

Let us consider a fully contracted term: we call such a term a big graph. A big graph is decomposed in small graphs, and each small graph will be estimated by its Hilbert-Schmidt norm, see [7]. A big graph is formed with:

vertices: the $\hat{g}_{i}, \hat{h}_{k}$, and $f_{i}$ functions and also the functions $\chi_{\Delta}$,

boson propagators noted $\sim$ and "propagators" noted $\leftarrow$.

The small graphs are:

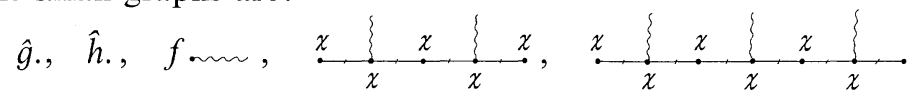

and also: $\phi b_{\text {reg }} \phi$ :

We first apply the H.S. norm to the vertices in $\hat{g}$ and $\hat{\mathrm{h}}$, this gives a bound:

$\prod_{j=1}^{N}\left(\|\hat{g}\|_{L^{2}}\|\hat{h}\|_{L^{2}}\right)^{4} \quad \mid$ big graph $\left.\right|^{1 / 2}$. 
To bound this new big graph, we first use the technique of $[7,9]$ and we extract from each "propagator" and each boson propagator localized in $\Delta$ and $\Delta^{\prime}$ a factor $e^{-O(1) d\left(\Delta, \Delta^{\prime}\right)}$ where $O(1)$ is taken as large as we want by taking $m$ large enough.

We then use the following bounds:

$$
\left|\tilde{\chi}_{\Delta}(p)\right| \leqq O(1) F(p), \quad \frac{1}{\left(p^{2}+m^{2}\right)} \leqq O(1) F(p)^{1-\varepsilon / 2} m^{-\varepsilon} \leqq e^{-Q_{8}} F(p)^{1-\varepsilon / 2}
$$

[for any $0<\varepsilon<1 / 3$ if $m$ is taken large enough depending on $Q_{8}$ ], and

$$
\frac{1}{\left(p^{2}+m^{2}\right)^{1 / 4}} \leqq O(1),\left|\left(p^{2}+m^{2}\right)^{-1 / 4} \frac{\not p+m}{\left(p^{2}+m^{2}\right)^{1 / 2}}\right| \leqq O(1) F(p)^{1 / 4-\varepsilon / 4} e^{-Q_{8} / 2} .
$$

Finally if a boson propagator is attached to a $f_{i}$-function of formula (I.2) we use $\frac{1}{p^{2}+m^{2}} \leqq \frac{1}{p^{2}+1} e^{Q_{8}} e^{-Q_{8}}$. The bounds for the small graphs are:

$$
\begin{aligned}
& \|m m\|_{\text {H.S. }}=|m m m|^{1 / 2} \leqq\|f\|_{-1} e^{Q_{8}} e^{-Q_{8}}
\end{aligned}
$$

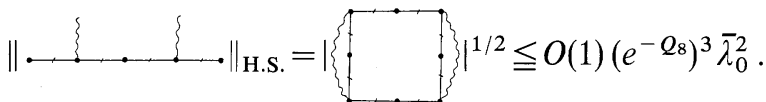

For the small graph with 3 boson lines, because the boson line can possibly contract between themselves we are obliged to consider:

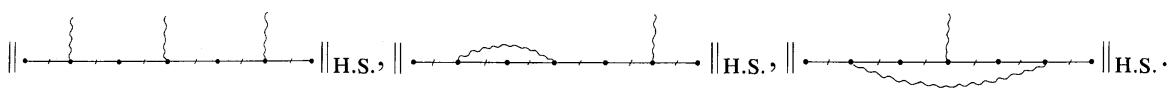

These 3 norms are bounded by $O(1)\left(e^{-Q_{8}}\right)^{3+1+\frac{1}{2}} \bar{\lambda}_{0}^{3}$.

Finally if $\Delta$ and $\Delta^{\prime}$ are neighbours or identic it is proved in [14] see also [16] that:

$$
\left\|\int \chi_{\Delta}: \phi b_{\text {reg }} \phi: \chi_{\Delta^{\prime}}\right\|_{\text {H.S. }} \leqq O(1) m^{-3 \varepsilon} \bar{\lambda}_{0}^{2} \leqq O(1)\left(e^{-Q_{8}}\right)^{3} \bar{\lambda}_{0}^{2} .
$$

If $\Delta$ and $\Delta^{\prime}$ have no intersection then it is trivial that:

$$
\left|b_{\text {reg }}(x, y) \chi_{\Delta}(x) \chi_{\Delta^{\prime}}(y)\right| \leqq O(1) e^{-m d\left(\Delta, \Delta^{\prime}\right) / 2} m^{-2 \varepsilon} \bar{\lambda}_{0}^{2} .
$$

From that we get:

$$
\left\|\chi_{\Delta}: \phi b_{\text {reg }} \phi: \chi_{\Delta^{\prime}}\right\|_{\text {H.S. }} \leqq O(1)\left(e^{-Q_{8}}\right)^{3} \bar{\lambda}_{0}^{2} e^{-m d\left(\Delta, \Delta^{\prime}\right) / 2} .
$$

We have then obtained for each term a bound consisting of:

Exponentially decreasing factors for all the localizations, and the decrease is as strong as we want provided that we take $m$ large enough.

A product of norm of small graphs, and for each term the number of small graphs is smaller than $O(1) L$ non counting the small graphs associated with $\hat{g}$, $\hat{h}$ and $f$.

So that each contracted terms is bounded by:

$$
\left.O(1)^{L}\left|e^{-Q_{8} \mid} \bar{\lambda}_{0}^{3 L}\right| e^{Q_{8}}\right|^{n} \prod_{i=1}^{n}\left\|f_{i}\right\|_{-1} \prod_{j=1}^{N}\left\|\hat{g}_{j}\right\|_{L^{2}}\left\|\hat{h}_{j}\right\|_{L^{2}} \prod e^{-O(1) d} .
$$


Thus collecting the various bound and taking $O(1) e^{-Q_{8}}=e^{-Q_{5}}$ we have:

$$
\begin{aligned}
& O(1)^{L}\left|\int R d \mu\right| \leqq O(1)^{N}\left(O(1) e^{Q_{5}}\right)^{n} \prod_{\Delta}(n(\Delta) !)^{1 / 2}\left(e^{-Q_{5}}\right)^{L} \bar{\lambda}_{0}^{3 L} e^{Q_{6}|\Gamma|} \\
& \prod_{\text {derivations }} e^{O(1)\left[d\left(\Delta_{j}, b_{j}\right)+d\left(\Delta_{j}^{\prime}, b_{j}\right)\right]} \prod_{i=1}^{n}\left\|f_{i}\right\|_{-1} \prod_{j=1}^{N}\left\|\hat{g}_{j}\right\|_{L^{2}}\left\|\hat{h}_{j}\right\|_{L^{2}} \prod_{\text {"propagators" }} e^{-O(1) d} .
\end{aligned}
$$

This finishes the proof og Lemma III.3.

\section{References}

1. Brydges, D.: Boundedness below for fermion model theories. Part I. J. Math. Phys. 16, 1649-1661 (1975); Part II. Commun. math. Phys. 47, 1-24 (1976)

2. Brydges, D., Federbush,P.: A semi-euclidean approach to Boson-Fermion model theories. J. Math. Phys. 15, 730-732 (1974)

3. Eckmann, J.P., Magnen, J., Seneor, R.: Decay properties and Borel summability for the Schwinger functions in $P(\phi)_{2}$ theories. Commun. math. Phys. 39, 4 (1975)

4. Glimm, J.: Yukawa coupling of quantum fields in two dimensions. Commun. math. Phys. 5, $343-386(1967) ; 6,61-76(1967)$

5. Glimm, J., Jaffe, A.: Self adjointness of the Yukawa ${ }_{2}$ Hamiltonian. Ann. Phys. 60, 321-383 (1970)

6. Glimm, J., Jaffe, A.: The Yukawa ${ }_{2}$ field theory without cutoffs. J. Funct. Anal. 7, 323-357 (1971)

7. Glimm, J., Jaffe, A.: Positivity of the $\phi_{3}^{4}$ Hamiltonian. Fortschr. Physik 21, 327-376 (1973)

8. Glimm, J., Jaffe, A., Spencer,T.: The cluster expansion. In: Constructive quantum field theory. Lectures Notes in Physics 25. Berlin-Heidelberg-New York: Springer 1973

9. Magnen,J., Seneor, R.: The infinite volume limit of the $\phi_{3}^{4}$ model. Ann. Inst. H. Poincare 24, 95-159 (1976)

10. McBryan, O.: Volume dependence of Schwinger functions in the Yukawa ${ }_{2}$ quantum field theory. Commun. math. Phys. 45, 279-294 (1975)

11. McBryan, O.: Finite mass renormalizations in the Yukawa 2 quantum field theory. Commun. math. Phys. 44, 237-243 (1975)

12. Schrader, R.: Yukawa quantum field theory in two space time dimensions without cutoffs. Ann. Phys. 70, 412-457 (1972)

13. Seiler, E.: Schwinger functions for the Yukawa model in two dimensions with space time cutoff. Commun. math. Phys. 42, 163-182 (1975)

14. Seiler, E., Simon, B.: Bounds in the Yukawa 2 quantum field theory. Commun. math. Phys. 45, 99-114 (1975)

15. Spencer,T.: The mass gap for the $P(\phi)_{2}$ quantum field model with a strong external field, A correction. Commun. math. Phys. 39, 75-76 (1974)

16. Magnen, J., Seneor,R.: The Wightman axioms for the weakly coupled Yukawa model in two dimensions. Preprint de l'Ecole Polytechnique, Octobre 1975

17. Cooper, A., Rosen, L.: The weakly coupled Yukawa ${ }_{2}$ field theory: cluster expansion and Wightman Axioms, University of Toronto preprint (1976)

Communicated by A. S. Wightman

Received March 8, 1976 
